\title{
Laser-induced self-assembly of quantum dots: deterministic case versus Brownian case
}

\author{
Aleksey SergeeVich TSipotan ${ }^{1^{*}}$, Viktor Aleksandrovich \\ TKachenko ${ }^{2}$, Aleksandr Sergeevich Aleksandrovsky ${ }^{1,3}$, Vitaliy \\ VASILYEVICH SLABKO ${ }^{1}$ \\ ${ }^{1}$ Institute of Engineering Physics and Radio Electronics, Siberian Federal University, 79 Svobodny \\ prospect, Krasnoyarsk, 660041, Russian Federation \\ ${ }^{2}$ Polytechnic Institute, Siberian Federal University, 79 Svobodny prospect, Krasnoyarsk, 660041, \\ Russian Federation \\ ${ }^{3}$ Laboratory of Coherent Optics, Kirensky Institute of Physics Federal Research Center KSC SB RAS, \\ Akademgorodok 50 bld.38, 660036 Krasnoyarsk, Russia \\ *acipotan@sfu-kras.ru
}

\begin{abstract}
The process of aggregation of two colloidal quantum dots in the field of quasiresonant laser radiation is investigated via computer simulation. The cases of either completely deterministic movement of particles or the movement with the account for random forces are considered. The conditions of pair formation are determined in dependence on initial interparticle distance, on laser wavelength, and on their initial orientation with respect to polarization plane of laser radiation. It is shown that despite velocity of thermal motion dominates over the velocity of directional diffusion of quantum dots, the account for random forces weakly influences on the probability of pair formation, the latter being close to results obtained in the deterministic case. The reason for this weak influence of Brownian movement is the resonant increase of electrodynamic attraction force during certain time interval within the laser pulse.
\end{abstract}

(c) 2018 Optical Society of America

Action of optical radiation onto nanoparticles leads to appearance of induced ac polarization on them that result in several effects. If we consider nanoparticles within tightly focused laser beam with its strong gradient of electric field strength, the interaction of induced polarization with external field leads to optical trapping when particles tend to the point when the field reaches maximum, and the interaction energy reaches minimum [1-5]. Optical trapping is feasible for obtaining aggregates of metal particles, for surface-enhanced Raman scattering molecular spectroscopy, for sorting of particles etc. [5]. Different approaches are suggested for enhancement of quality of optical trapping. Thus, in [6] ultrasmooth gold nanoparticles were synthesized and two-fold and ten-fold reduction in standard deviation for trap stiffness measurements was observed in liquid and air, respectively. Analytical calculation [7] shows that trapping strength and trapping efficiency for hollow metal nanoparticles are much better than for solid ones. Another approach is based on the creation of larger gradient of external field e.g. by using planar silicon metalenses as the focusing element [8]. The conditions for optical trapping can be implemented by the interfering beams [9] or highly focused radially polarized beams [10].

Another case of interaction between the external field and nanoparticles is the optical binding that generally arises from the interaction between ac polarizationjs induced on the nanoparticles by external radiation field [11]. Large attention is attracted by the case of optical binding of metal nanoparticles possessing plasmon resonance that can be used for amplification of the interaction induced by external optical field [12-14] and for formation of structures with dimensions much smaller than the wavelength of external field. The authors of [15] have shown that the presence of metal surface in the vicinity of interacting pair of particles additionally allows to enhance optical binding. The minimum of energy of induced 
dipole-dipole interaction is not much more than the energy of thermal movement at the radiation parameters close to the radiation stiffness threshold of metal nanoparticles. Optical binding can be used for the formation of nanostructures not only from metal particles but from semiconducting ones, e.g., optical binding of silicon nanowires in counter-propagating beams was demonstrated [16]. Colloidal quantum dots (QDs) possessing narrow-bandwidth excitonic transitions can form structures with pre-defined geometry with the help of optical binding, as well. Calculations [17] have shown that the energy of induced dipole-dipole interaction between QDs exceeds that of interaction between metal nanoparticles by several orders of magnitude. The energy of interparticle interaction in the field of laser radiation depends on its frequency, on resonant frequencies of particles and on the orientation of the group of particles with respect to the polarization plane or radiation, that can be the base of selective formation of various nanostructures with pre-defined shape and content.

Large excess of potential well depth in case of QDs invokes that optical binding may not only form structures that are stable during the period of action of external field but to overcome the potential barrier surrounding individual particles and to form structures that will be stable after radiation is switched off.

The results of experimental formation of stable pairs of colloidal QDs of CdTe after the exposure by quasi-resonant laser radiation [18] demonstrate correctness of the above assumptions and stimulate the formulation of several tasks for a computer simulation that are important for understanding the essence of the processes of structures assembly. In the particular, in experiment [18] a colloidal solution was used with the random distribution of distances between QDs and with random orientation of interacting pairs with respect to the laser radiation polarization plane. Factors affecting the dynamics of colloidal QDs and their subsequent aggregation are electrostatic repulsion force that prevents from spontaneous aggregation, van der Waals force ensuring long-term stability of structure obtained, viscous friction force, stochastic hydrodynamical force, and electrodynamical interaction forces between induced dipoles

In the present study, on the base of computer simulation, the analysis of effect of Brownian movement of the particles in comparison with deterministic movement is done. In both cases the influence of QDs concentration and laser wavelength on the aggregation of particles is investigated.

\section{Nanostructures formation model}

For the description of the dynamics of self-organization of particles in the field of laser radiation we use the elements of the physical model proposed in [19]. Let us consider the ensemble of $N$ particles with the mass $m_{j}$, radius $R_{j}$ and resonant frequency $\omega_{j}^{0}, j=1, \ldots, N$, in the field of laser radiation. For every $j$-th particle and for every time moment $t$ we define following quantities:

$\vec{r}_{j}=\left(r_{j}^{x}, r_{j}^{y}, r_{j}^{z}\right)^{T}$ are the radius-vector of the mass center and its projections;

$\vec{V}_{j}=\left(V_{j}^{x}, V_{j}^{y}, V_{j}^{z}\right)^{T}$ are the vector of translational velocity of the mass center and its projections;

$\vec{d}_{j}=\left(d_{j}{ }^{x}, d_{j}{ }^{y}, d_{j}{ }^{z}\right)^{T}$ are the vector of field-induced dipole moment and its projections.

Let us assume that the ensemble dimensions are much smaller than the wavelength of external radiation that will allow external light field $\vec{E}$ to be considered homogeneous in all points of ensemble, so that retardation effect could be neglected [14]. Then electric field vector of the light wave can be presented in the form of harmonic function oscillating only in time with the frequency $\omega, \vec{E}=1 / 2 \vec{E}_{0} \exp (i \omega t)+c . c$. 
Local field in the vicinity of $\mathrm{j}$-th particle contains contributions from external field and from all other particles that is accounted for by summing the fields of all dipoles induced on them. Thus, dipole moment vector induced on $\mathrm{j}$-th particle equals to

$$
\vec{d}_{j}=\chi_{0 j}\left(\vec{E}+\sum_{k \neq j} \vec{E}_{k}\right)
$$

where $\chi_{0 j}$ is the linear polarizability of isolated $\mathrm{j}$-th particle, $\vec{E}_{k}$ is the field produced by $k$-th particle of ensemble in the point where $\mathrm{j}$-th particle is positioned:

$\vec{E}_{k}=\frac{1}{4 \pi \varepsilon_{0}} \frac{3\left(\vec{d}_{k}, \vec{r}_{j k}\right) \vec{r}_{j k}-\vec{d}_{k}\left|r_{j k}\right|^{2}}{\left|r_{j k}\right|^{5}}$,

where $\vec{r}_{j k}=\vec{r}_{j}-\vec{r}_{k}, \varepsilon_{0}=(36 \pi)^{-1} \cdot 10^{-9} C^{2} /\left(N \cdot m^{2}\right)$ is the electric constant.

According to [13] and using Eq. (2) we obtain the expression describing the energy of $j$-th dipole:

$W_{j}^{d}=\frac{1}{4 \pi \varepsilon_{0}} \sum_{k \neq j} \frac{\left(\vec{d}_{j}, \vec{d}_{k}\right)\left|\vec{r}_{j k}\right|^{2}-3\left(\vec{d}_{j}, \vec{r}_{j k}\right)\left(\vec{d}_{k}, \vec{r}_{j k}\right)}{\left|\vec{r}_{j k}\right|^{5}}$

Dipolar polarizability of the particles will be described in the frame of two-level model. In this simplest case of a single resonance at the frequency $\omega^{r}$, characterized by electric dipole moment of the transition $\left|d_{12}\right|^{2}$ and by relaxation rate (homogeneous width) $\Gamma$ dipole polarizability of particle in the vicinity of $\omega^{r}$ can be described as follows:

$\chi=\frac{\left|d_{12}\right|^{2}}{\hbar(\Omega+i \Gamma)}$

where $\Omega=\omega^{r}-\omega$ is the detuning from resonance and $\hbar$ is Planck constant.

Calculating the overall energy of pair interaction, one must take into account the van der Waals energy and the energy of electrostatic repulsion, the expressions for those being given in $[20,21]$. Parameters of the medium and of the field are assumed to be chosen so that electrostatic repulsion force exceeds van der Waals force while action of laser radiation would lead to the formation of exclusively resonant structures.

Translational movement of $\mathrm{j}$-th particle $(\mathrm{j}=1,2)$ we describe by Langevin equation system

$$
\left\{\begin{array}{l}
d \vec{r}_{j} / d t=\vec{v}_{j} \\
m_{j}\left(d \vec{v}_{j} / d t\right)=\vec{F}_{j}^{v}+\vec{F}_{j}^{e}+\vec{F}_{j}^{d}-\vec{F}_{j}^{f}+\vec{F}_{j}^{c}
\end{array}\right.
$$

where $\vec{v}_{j}$ is the velocity of $\mathrm{j}$-th particle, $\vec{F}_{j}^{d}=-\operatorname{grad}\left(W_{j}^{d}\right)$ is the electrodynamical force, $\vec{F}_{j}^{v}=-\operatorname{grad}\left(W_{j}^{v}\right)$ is van der Waals force, $\vec{F}_{j}^{e}=-\operatorname{grad}\left(W_{j}^{e}\right)$ is the electrostatic repulsion force; $\vec{F}_{j}^{f}-$ is the viscous friction force, $\vec{F}_{j}^{c}$ - is stochastic hydrodynamical force.

Viscous friction force, being main factor of kinetic energy dissipation, for spherical particles with the radius $R_{j}$ is determined by Stokes formula

$\vec{F}_{j}^{f}=6 \pi \eta R_{j} \vec{v}_{j}$

where $\eta$ is dynamic viscosity of the medium. 
Interaction of particles with the environment having fluctuating density leads to random change of their movement trajectory that must compensate for the action of friction force and preserve the mobility of dispersed phase. For modeling of Brownian motion we assume that random force $\vec{F}_{j}^{c}$ is governed by Gauss distribution. Before every integration step the values of projections of random force $\left(\vec{F}_{i}^{c}\right)^{k}(k=x, y, z)$ onto the coordinate axes are chosen from Gaussian distribution with zero mean value and the deviation equal to

$\delta^{2}=\frac{12 \pi \eta R_{j} k_{b} T}{\Delta t}$

here $k_{b}$-is the Boltzmann constant, $T$-is the temperature of the medium.

Consider the case of interaction of a pair of identical QDs in homogeneous ac external field $\vec{E}$. Calculation of van der Waals force $\vec{F}_{j}^{v}$ and of electrostatic force is rather easy and is omitted here [20]. Expression for electrodynamical force averaged over the field oscillation period that acts onto the first particle $\left\langle\vec{F}^{d}\right\rangle$ has the form of four terms (the force acting on the second particle is $\left.-\left\langle\vec{F}^{d}\right\rangle\right)$ :

$\left\langle\vec{F}^{d}\right\rangle=\left\langle\vec{F}_{\mathrm{I}}^{d}+\vec{F}_{\mathrm{II}}^{d}+\vec{F}_{\mathrm{III}}^{d}+\vec{F}_{\mathrm{IV}}^{d}\right\rangle$

where $\quad \vec{F}_{\mathrm{I}}^{d}=\frac{3}{4 r^{5}}\left[\left[\vec{r} \times \vec{d}_{1}\right] \times \vec{d}_{2}\right], \quad \vec{F}_{\mathrm{II}}^{d}=\frac{3}{4 r^{5}}\left[\left[\vec{r} \times \vec{d}_{2}\right] \times \vec{d}_{1}\right], \quad \vec{F}_{\mathrm{III}}^{d}=-\frac{6}{4 r^{5}} \vec{r}\left(\vec{d}_{1} \cdot \vec{d}_{2}\right)$, $\vec{F}_{\mathrm{IV}}^{d}=\frac{15}{4 r^{7}} \vec{r}\left(\left[\vec{r} \times \vec{d}_{1}\right] \cdot\left[\vec{r} \times \vec{d}_{2}\right]\right), \vec{r}-$ is the vector connecting the centers of the first and the second particles, $\vec{d}_{1}, \vec{d}_{2}$ are the field-induced dipole moments onto particles 1 and 2 , correspondingly. For analysis of components of electrodynamical force we introduce the coordinate system in which the particles are positioned in XY plane, the field $\vec{E}$ is directed along $\mathrm{X}$ axis (Fig. 1) and the angle $\theta$ is the angle of deviation that determines the mutual position of the particles with respect to the field vector.

The terms $\vec{F}_{\text {III }}^{d}$ и $\vec{F}_{\text {IV }}^{d}$ are central forces while $\vec{F}_{\mathrm{I}}^{d}$ and $\vec{F}_{\mathrm{II}}^{d}$ are non-central ones, and in the case of two identical particles under consideration $\vec{F}_{\mathrm{I}}^{d}=\vec{F}_{\mathrm{II}}^{d}=\vec{F}_{\mathrm{I}, \mathrm{II}}^{d}$. In the coordinate system introduced above from Eq. (1), (2) and (4) the expressions for components of induced dipole moment vector can be obtained:

$$
\begin{aligned}
& d_{x}=\omega_{s}|r|\left(\frac{\sin ^{2}(\theta)}{\left(\Omega+\omega_{s}\right)+i \Gamma}+\frac{\cos ^{2}(\theta)}{\left(\Omega-2 \omega_{s}\right)+i \Gamma}\right)\left|\frac{\vec{E}_{0}}{2}\right| e^{i \omega t}+c . c . \\
& d_{y}=\omega_{s}|r|\left(\frac{\cos (\theta) \sin (\theta)}{\left(\Omega+\omega_{s}\right)+i \Gamma}-\frac{\cos (\theta) \sin (\theta)}{\left(\Omega-2 \omega_{s}\right)+i \Gamma}\right)\left|\frac{\vec{E}_{0}}{2}\right| e^{i \omega t}+c . c .
\end{aligned}
$$

Here $\omega_{s}=|d|^{2} / \hbar r^{3}$ is the shift of resonance stipulated by dipole-dipole interparticle interaction. If radius vector connecting particles' centers $\vec{r}$ (Fig. 1) is collinear to electric field vector of external light wave $\vec{E}(\sin (\theta)=0)$, the resonant frequency is shifted to the red part of spectrum by the value $2 \omega_{s}$. In case of orthogonal orientation of $\vec{r}$ vector with respect to $\vec{E}(\cos (\theta)=0)$ resonant frequency experiences shift to the blue part of spectrum by $\omega_{s}$.

From the expression for dipole moments Eq. (9) the conclusions of directions of components of electrodynamical force Eq.(8) can be done. Consider the components of this 
force acting from the second particle onto the first one (the same components acting onto the second particle are directed oppositely). Components of $\vec{F}_{\text {III }}^{d}$ и $\vec{F}_{\text {IV }}^{d}$ are central ones and always directed along radius vector $\vec{r}$. Herein $\vec{F}_{\text {III }}^{d}$ corresponds to attraction between particles (i. e., directed from second particle to the first one), $\vec{F}_{\mathrm{IV}}^{d}$ corresponds to repulsion (directed from first particle to second one). Analysis shows that forces $\vec{F}_{\mathrm{I}, \mathrm{I}}^{d}$ are non-central ones and always lie in XY plane (Fig. 1), their direction and magnitude being determined by distance $\vec{r}$, deviation angle $\theta$ and ratios of parameters $\Gamma,\left|d_{12}\right|^{2}, \vec{r}$ and $\Omega$, and it follows from the properties of triple vector product that they always have a projection on the direction of particles' attraction.

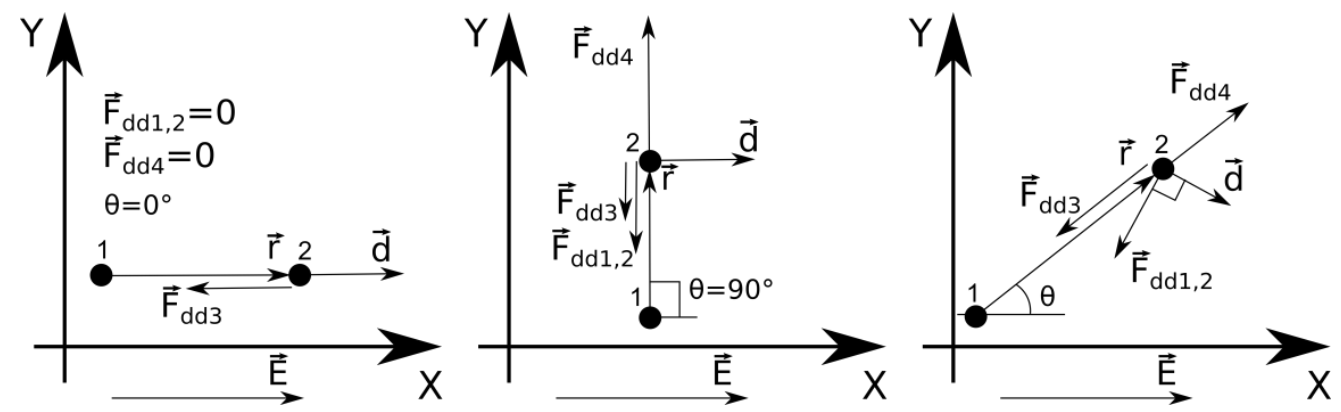

Fig. 1 Diagram of directions of components of electrodynamical force.

Modeling of laser-stimulated aggregation of pair of particles into a stable structure with the help of Brownian dynamics with the account of all forces acting onto the particles allows estimating parameters of medium and field necessary for the experiment. Analysis of expressions for electrodynamical forces does not allow even qualitative description of system behavior since distance, angle $\theta_{0}$ and $\omega_{s}$ are continuously changing in the motion process. In the general case the integration of system Eq. (5) can be done only numerically.

\section{Computer simulation}

Account for random force allows describing Brownian motion of particles, however, it however, it greatly complicates the calculations. We conducted computer simulations both without account and with account for the random force that allows analysis of influence of Brownian motion on the process of self-assembly.

In the first case $\left(\vec{F}_{j}^{c}=0\right)$ two QDs were placed at certain distance $\left(r_{0}\right)$ from each other in the XY plane on the axis oriented at certain deviation angle $\theta_{0}$. According to estimates [22], starting parameters were chosen as follows: electric field strength vector of laser radiation is directed along $\mathrm{X}$ axis, the electric field strength magnitude $\mathrm{E}=7000 \mathrm{ESU}$. Radius of QDs 1.5 $\mathrm{nm}$, density of QDs $5.81 \mathrm{~g} / \mathrm{cm}^{2}, \eta=0.8902 \mathrm{mPa} \cdot \mathrm{sec}$, absorption line width $\Delta \lambda=15 \mathrm{~nm}$, resonant wavelength изолированной QDs $\lambda_{\text {rez }}=525 \mathrm{~nm}$, irradiation time corresponding to laser pulse duration $10 \mathrm{~ns}$.

Earlier calculations and experiments have shown that structure formation probability depends on the external radiation wavelength, being the parameter of importance. Therefore, in present simulations the dependence of aggregation probability of pair of particles averaged on the angle $\theta_{0}$ was investigated on the external radiation wavelength. Step-to-step method was used to determine the force of interaction of the particles with account for friction, van der Waals force, electrostatic repulsion and mutual influence of dipole moments, and the 
velocity and displacement during given pulse duration (10 ns) were calculated. Angle $\theta_{0}$ was varied from $0^{\circ}$ to $90^{\circ}$ with a step $1^{\circ}$. Laser wavelength was varied from 450 to $950 \mathrm{~nm}$ with a sep $5 \mathrm{~nm}$. Simulations were done for three initial distances $r_{0}=10 \mathrm{~nm}, r_{0}=16 \mathrm{~nm}, r_{0}=20$ $\mathrm{nm}$.

Figure 2(a) presents the dependence of angle $\theta_{0}$ averaged probability of aggregation of pair of particles on the laser wavelength $P^{*}(\lambda)$ for different initial distances $r_{0}$. This probability can take values either 0 or 1 , in dependence on the combination of initial distance and angle. Value $P^{*}(\lambda)=0$ corresponds to the combination of parameters at which aggregation does not happen, and oppositely, $P^{*}(\lambda)=1$ corresponds to the aggregation. After averaging over all initial angles probability can take any intermediate value between 0 and 1 . Maximum aggregation probability (indicated by points in the graph) is attained at the wavelengths $\lambda_{\text {mp }}\left(r_{0}=16 n m\right)=640 \mathrm{~nm}, \lambda_{\text {mp }}\left(r_{0}=20 \mathrm{~nm}\right)=580 \mathrm{~nm}$. For $r_{0}=10 \mathrm{~nm} \lambda_{\mathrm{mp}}$ was not determined $\left(\lambda_{\mathrm{mp}}\left(r_{0}=10 \mathrm{~nm}\right)>950 \mathrm{~nm}\right)$. Figure 2 (b) presents spectral dependence of mean aggregation time $t_{\mathrm{av}}^{*}(\lambda)$. Minimum aggregation time (indicated by points in the graph) is attained at wavelengths $\quad \lambda_{\mathrm{mt}}\left(r_{0}=10 \mathrm{~nm}\right)=660 \mathrm{~nm} \quad, \quad \lambda_{\mathrm{mt}}\left(r_{0}=16 \mathrm{~nm}\right)=560 \mathrm{~nm} \quad$ ， $\lambda_{\mathrm{mt}}\left(r_{0}=20 \mathrm{~nm}\right)=540 \mathrm{~nm}$.

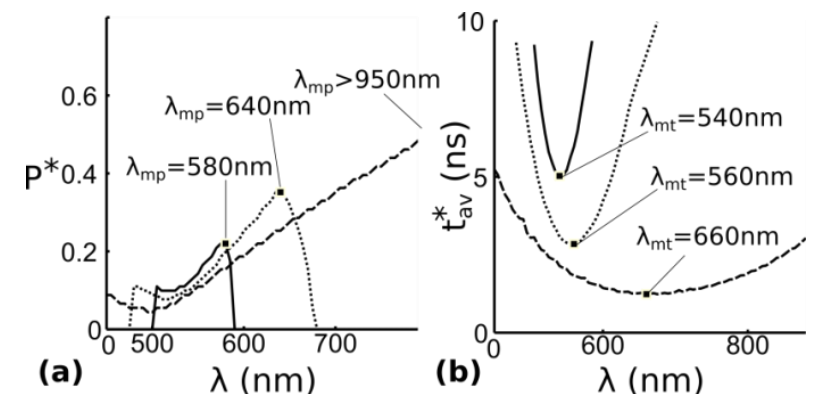

Fig 2. (a) Dependences of probability of sticking of a pair of particles $P^{*}(\lambda)$ on the laser radiation wavelength. (b) Dependence of average sticking time of pair of particles $t_{\mathrm{av}}^{*}(\lambda)$ on the laser radiation wavelength.

As one can see from Fig. 2(a) for distances $r_{0}$ equal to 20 and $16 \mathrm{~nm}$ the dependence of aggregation probability on the external field wavelength is observed. Decrease of initial distance $r_{0}$ leads to the increase of probability and broadening of the spectral range of possible aggregation, and maximum of aggregation probability becomes shifted to the red part of spectrum. Really, in accordance with Eq.(9), decrease of $r_{0}$ leads to increase of both offset frequency $\omega_{s}$ and attraction force. $\vec{F}_{\text {III }}^{d}$ for a pair of those particles for which the radius vector connecting their centers is oriented along $X$ axis. Decrease of aggregation time (Fig. 3(b)) is due to the same cause. Herein, other components of electrodynamical force (repulsion and non-central forces) that depend on angles $\theta_{0}$ experience increase, too. In this regard, it is obvious that probability of aggregation must depend on the initial angle $\theta_{0}$.

To prove this assumption let us consider the trajectories of deterministic movement of particles for 5 different situations in dependence of initial angle $\theta_{0}: I . r_{0}=16 \mathrm{~nm}, \lambda=640 \mathrm{~nm}$; II. $r_{0}=20 \mathrm{~nm}, \lambda=580 \mathrm{~nm}$; III. $r_{0}=10 \mathrm{~nm}, \lambda=525 \mathrm{~nm}$; IV. $r_{0}=16 \mathrm{~nm}, \lambda=525 \mathrm{~nm} ;$ V. $r_{0}=20 \mathrm{~nm}, \lambda=525 \mathrm{~nm}$. First two cases correspond to maximum aggregation probability for 
chosen distances. Three other cases describe irradiation of particles at the resonant wavelength of isolated particle for every chosen initial distance.

Figure 3 presents some trajectories obtained for 5 different cases specified above. In cases I and II trajectories corresponding to initial angles $\theta_{0}<\theta_{\lim }$ (threshold angle $\theta_{\lim }$ is marked by dashed line in the graphs) lead to collision and aggregation of particles. Trajectories corresponding to angles $\theta_{0}>\theta_{\lim }$ do not lead to particles aggregation. Therefore, in the deterministic case the possibility of particles collision depends on the initial deviation angle, and all particles that do not satisfy threshold condition do not aggregate. Consequently, to increase the aggregation probability the irradiation wavelength must be chosen to be close to the resonance of interacting particles system but not to the resonance of isolated particle.
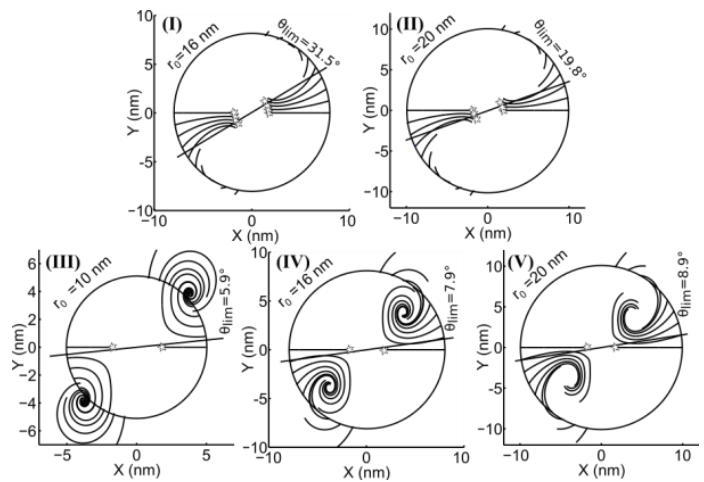

Fig. 3. Trajectories of particles (solid lines) for five different cases specified in the text. Points of particles' collisions are marked by asterisks.

Figure 4 depicts temporal dependences of particles' velocities normalized by root mean square $(\mathrm{rms})$ thermal velocity $\left(\sqrt{\left\langle v_{T}^{2}\right\rangle}=\sqrt{3 k_{\mathrm{b}} T / m} \approx 1181 \mathrm{~cm} / \mathrm{s}\right.$ ) for all three wavelengths under consideration.
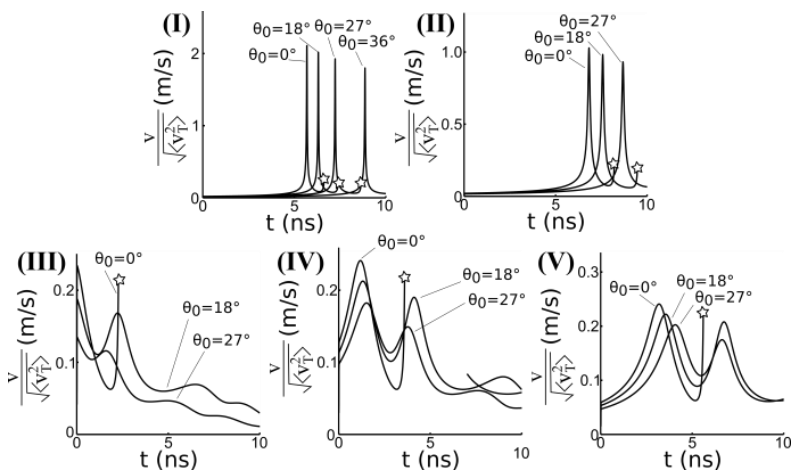

Fig. 4. Dependence of modulus of quantum dots velocity normalized by rms thermal velocity on the duration of action of laser radiation, for several initial angles $\theta_{0}$.

For chosen parameters of the medium and the field, in all cases the velocity of directional diffusion is much smaller than thermal movement velocity except for some time intervals in cases I and II. Latter is connected with the fact that directional motion leads to decrease of interparticle distance and consequently, to change of $\omega_{s}(t)$. At certain time period laser wavelength falls into the resonance with $\omega_{s}(t)$ resulting in the resonant increase of attraction force (Eq. 8 and 9). Further consequence is the increase of velocity followed by its decrease 
associated with overcoming a potential barrier. Subsequent velocity increase until the collision is due to upraise of van der Waals force.

Therefore, deterministic case is extremely useful for clarifying the details of electrodynamic interaction, but one must expect that it will be rather impractical for prognosis of real aggregation probabilities. In reality the influence of stochastic factor on the trajectories of particle motion must be important. However, results of calculations with the account for stochastic force that will be presented below demonstrate that it is not exactly right.

Let us determine the conditions of pair formation and aggregation probability with the account for Brownian motion for cases I and II. Account for random force $\vec{F}_{j}^{c}$ leads to the necessity to use 3D model [22] since Brownian motion can withdraw particles off the XY plane. Brownian motion is probabilistic, and modeling was conducted via averaging over 100 implementations for every case of starting positions. Figure 5 depicts the dependence of aggregation probability on initial angle for all 5 cases.
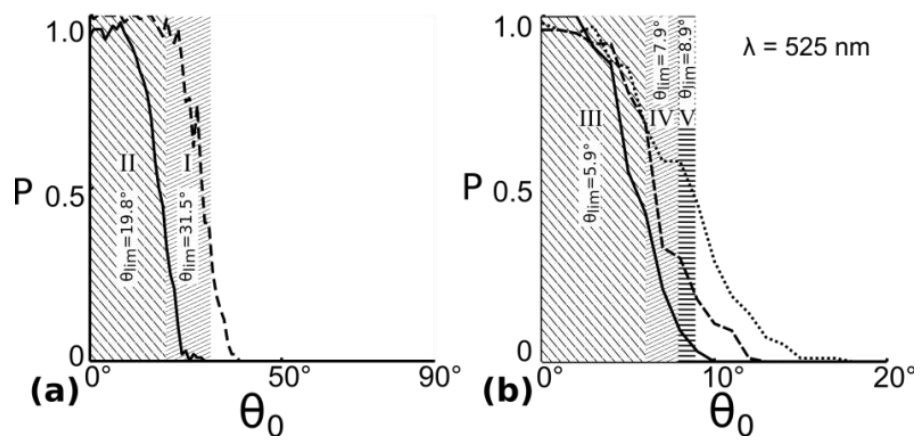

Fig.5. Dependence of aggregation probability on the initial angle $\theta_{0}$ for 5 cases specified in the text, with the account for Brownian motion (solid lines). Shaded areas indicate 5 different cases for the model that does not take thermal motion into account.

Figure 5 shows that account for Brownian motion results in nonzero probability of collision of particles with initial angle above $\theta_{\lim }$ and in decrease of probability for smaller angles. On the whole, introduction of Brownian motion decreases integral probability approximately by $2 \%$ for cases I and II and by less than $1 \%$ for cases III, IV, V.

\section{Conclusion}

The self-organized pair formation from isolated colloidal quantum dots in the field of quasiresonant laser radiation is studied via computer simulation. The model is based on account for van der Waals forces, electrostatic repulsion forces, viscosity friction, stochastic hydrodynamical force and electrodynamical force. The influence of initial distances between a pair of nanoparticles $r_{0}$, of their initial orientation angle $\theta_{0}$ with respect to laser radiation polarization plane, and of the external field wavelength on the aggregation probability is investigated. It was shown that under certain parameters of the medium (viscosity and temperature) and of the field (intensity and pulse duration) aggregation probability is close to $100 \%$ under the condition $\theta_{0}<\theta_{\lim }$ when only deterministic forces are taken into account.

$\theta_{\lim }$ grows with the decrease of $r_{0}$, and the spectral range of laser wavelengths for which the aggregation is possible broadens Account for Brownian motion of particles does not change the nature of the dependence of aggregation probability on $r_{0}$, however, its magnitude decreases in the range $\theta_{0}<\theta_{\text {lim }}$ and becomes nonzero in the range $\theta_{0} \geq \theta_{\text {lim }}$.

Character of deterministic trajectories of motion within $\theta_{0}<\theta_{\lim }$ range is determined mainly by central forces and is close to linear one. Velocity of particles' movement increases 
when the resonant frequency of the system of two quantum dots falls into the resonance with laser wavelength, and upon decrease of interparticle distance. For $\theta_{0} \geq \theta_{\lim }$ the character of the particles' movement is determined by central forces with the increasing influence of noncentral ones. Velocities of particles' movement are generally smaller than thermal velocity and exceed it only within certain time periods for $\theta_{0}<\theta_{\lim }$. Therefore, particles' trajectories are correctly described only with the account for Brownian motion.

Oppositely, calculations of aggregation probability with account for Brownian motion and for deterministic case give very similar results. Therefore, for investigation of the influence of parameters of the medium and the field on aggregation probability, deterministic computer modeling can be used.

\section{Funding}

Russian Science Foundation (Grant 18-72-00003). V.V. Slabko is grateful for the support from the Ministry of Education and Science of the Russian Federation (Grant 3.6341.2017/VU).

\section{References}

1. T. A. Nieminen, N. du Preez-Wilkinson, A.B. Stilgoe, et.al., "Optical tweezers: Theory and modeling," J Quant Spectrosc Radiat Transf 146, 59-80 (2014).

2. P. Polimeno, A. Magazzù, M. A. Iatì, et.al., "Optical tweezers and their applications," J Quant Spectrosc Radiat Transf 218, 131-150 (2018).

3. S.E. S. Spesyvtseva, K. Dholakia, "Trapping in a material world," ACS Photonics 3 (5), 719-736 (2016).

4. C. Bradac, "Nanoscale optical trapping: A review," Adv. Optical Mater. 6, 1800005(2018).

5. A. Lehmuskero, P. Johansson, H. Rubinsztein-Dunlop, L. Tong, and M. Kall, "Laser trapping of colloidal metal nanoparticles," ACS Nano 9(4), 3453-3469( 2015).

6. Y. Arita, G. Tkachenko, N. McReynolds, et.a. "Optical trapping of ultrasmooth gold nanoparticles in liquid and air," APL Photonics 3, 070801 (2018).

7. E. Madadi “Optical trapping calculations for hollow metallic nanoparticles," J. Opt. Soc. Am. B 33 (4), 670-674 (2016).

8. G. Tkachenko, D. Stellinga, A. Ruskuc, M. Chen, K. Dholakia and T.F. Krauss, "Optical trapping with planar silicon metalenses," Opt. Lett. 43 (14), 3234-3227 (2018).

9. M. Mohammadnezhad, A. Hassanzadeh, "Evanescent field interferometric optical tweezers with rotational symmetric patterns," J. Opt. Soc. Am. B 34 (5), 983-990 (2017).

10. M. Li, S. Yan, B. Yao, M. Lei, Y. Yang, J. Min and D. Dan, "Trapping of Rayleigh spheroidal particles by highly focused radially polarized beams," J. Opt. Soc. Am. B 32 (3), 468-472 (2015).

11. K. Dholakia, P. Zemanek, “Gripped by light: Optical binding,” Rev. Mod. Phys. 82, 1787-1791(2010).

12. A. S. Zelenina, R. Quidant, and M. Nieto-Vesperinas, "Enhanced optical forces between coupled resonant metal nanoparticles," Opt. Lett. 32(9), 1156-1158 (2007).

13. S.V. Karpov, A.K. Popov, V.V. Slabko, "Photochromic reactions in silver nanocomposites with a fractal structure and their comparative characteristics," Technical Physics 48 (6), 749-756(2003).

14. V. V. Slabko, G. G. Khachatryan, and A. S. Aleksandrovsky, "Self-organized aggregation of small metal particles controlled by an external light field," JETP Lett. 84(6), 300-304 (2006).

15. N. Kostina, M. Petrov, A. Ivinskaya, et.al. "Optical binding via surface plasmon polariton interference," Phys. Rev. B 99, 125416 (2019).

16. M.G. Donato, O. Brzobohaty', S.H. Simpson, et.al. "Optical trapping, optical binding, and rotational dynamics of silicon nanowires in counter-propagating beams," Nano Lett. 19, 342-352 (2019).

17. V. V. Slabko, A. S. Tsipotan, and A. S. Aleksandrovsky, "Resonant light-controlled self-assembly of ordered nanostructures," Photon. Nanostruct. Fundam. Appl. 10(4), 636-643 (2012)

18. A. S. Tsipotan, M.A. Gerasimova, V.V. Slabko and A.S. Aleksandrovsky, "Laser-induced wavelengthcontrolled self-assembly of colloidal quasi-resonant quantum dots," Opt. Express 24(10), 11145-11150 (2016)

19. V. V. Slabko, A. S. Tsipotan, and A. S. Aleksandrovsky, "Self-organised aggregation of a pair of particles with different resonant frequencies and electric dipole moments of transitions, controlled by an external quasiresonant field," Quantum Electron. 43 (5), 458-462 (2013).

20. S.V. Karpov, I.L. Isaev, A.P. Gavrilyuk, et.al., "General principles of the crystallization of nanostructured disperse systems," Colloid Journal 71 (3), 313-328 (2009).

21. N. Jacob, "Intermolecular and surface forces", Academic Press, USA, P. 450 (1992).

22. V.A. Tkachenko, A.S. Tsipotan, A.S. Aleksandrovsky, V.V. Slabko, "Three-dimensional model of quantum dots' self-assembly under the action of laser radiation," Computer Optics 41 (4), 577-580 (2017). 